DOI: 10.17516/1997-1397-2021-14-4-425-432

УДК 517.9

\title{
Boundary Value Problems for Fourth-Order Sobolev Type Equations
}

\author{
Alexander I. Kozhanov* \\ Sobolev Institute of Mathematics \\ Novosibirsk, Russian Federation
}

Received 10.03.2021, received in revised form 05.04.2021, accepted 20.05.2021

\begin{abstract}
The goal of the article is the study of solvability in the Sobolev spaces of boundary value problems for some classes of Sobolev-type fourth-order linear equations. We will prove that an initial boundary value problems well problems with data both at the initial time moment and the final time moments can be well-posed for the equations under study.
\end{abstract}

Keywords: Sobolev-type fourth-order differential equation, boundary value problem, existence, uniqueness.

Citation: A. I. Kozhanov, Boundary Value Problems for Fourth-Order Sobolev Type Equations, J. Sib. Fed. Univ. Math. Phys., 2021, 14(4), 425-432. DOI: 10.17516/1997-1397-2021-14-4-425-432.

\section{Introduction}

The article is devoted to the study of the solvability of boundary value problems for differential equations

$$
D_{t}^{4} u+\sum_{k=0}^{3} A_{k} D_{t}^{k} u=f(x, t) \quad\left(D_{t}^{k}=\frac{\partial^{k}}{\partial t^{k}}, \quad k=\overline{0,4}\right)
$$

with operators $A_{k}$ of the form

$$
A_{k}=\frac{\partial}{\partial x_{i}}\left(a^{i j, k}(x) \frac{\partial}{\partial x_{j}}\right)+a_{0, k}(x)
$$

(here and below, summation over repeated indices from 1 to $n$ is carried out).

The differential equations $(*)$ are recently attributed to the class of Sobolev-type equations. Various aspects of the theory of Sobolev-type equations are reflected in monographs [1-7] and also in numerous journal articles (it is impossible to mention even a small part of such articles just because they are numerous).

For Sobolev-type differential equations, best studied is the solvability of the Cauchy problem and initial boundary value problems. At the same time, as is shown in $[3,8]$, in some case, for Sobolev-type equations, simultaneously with initial boundary value problems, other problems can also be well-posed; these include problems with data both at the initial and final time moments. In the present article, for equations $(*)$, we study the solvability both of initial boundary value problems and problems with data at different time moments.

Clarify that the goal of the present article is to prove the solvability of some problem for equations $(*)$ in the classes of regular solutions, i.e., solutions having all weak derivatives in the sense of Sobolev [9-11] occurring in the equation.

Formally, equation $(*)$ with the above operators is a fifth-order equation. The use of the term "fourth-order Sobolev equation" in the title and the article means that the equations under study

*kozhanov@math.nsc.ru

(C) Siberian Federal University. All rights reserved 
are fourth-order equations with respect to the time (distinguished) variable, which is the leading variable and defines the statements of the problems.

One more remark: Equations $(*)$ have model and the simplest form. We will speak of some more general equations and of generalizations of the results at the end of the article.

\section{Statements of the Problems}

Suppose that $\Omega$ is a bounded domain in $\mathbb{R}^{n}$ with smooth (for simplicity, infinitely differentiable) boundary $\Gamma, Q$ is the cylinder $\Omega \times(0, T)$ of finite height $T$, and $S=\Gamma \times(0, T)$ is the lateral boundary of $Q$. Furthermore, let $a^{i j, k}(x), a_{0, k}(x), i, j=1, \ldots, n, k=0, \ldots, 3, f(x, t)$ be given functions defined for $x \in \bar{\Omega}$ and $t \in[0, T]$ and let $A_{k}$ and $L$ be the differential operators whose action at a given function $v(x, t)$ is defined by the equalities

$$
\begin{gathered}
A_{k} v=\frac{\partial}{\partial x_{i}}\left(a^{i j, k}(x) v_{x_{j}}\right)+a_{0, k}(x) v, \\
L v=D_{t}^{k} v+\sum_{k=0}^{3} A_{k} D_{t}^{k} v .
\end{gathered}
$$

Boundary Value Problem I: Find a function $u(x, t)$ that is a solution to the equation

$$
L u=f(x, t)
$$

in the cylinder $Q$ such that

$$
\begin{gathered}
\left.u(x, t)\right|_{S}=0 \\
\left.D_{t}^{k} u(x, t)\right|_{t=0, x \in \Omega}=0, \quad k=0,1,2,3 .
\end{gathered}
$$

Boundary Value Problem II: Find a function $u(x, t)$ that is a solution to equation (1) in $Q$ and satisfies conditions (2) and also the condition

$$
\left.D_{t}^{k} u(x, t)\right|_{t=0, x \in \Omega}=0, \quad k=0,1,2,\left.\quad D_{t}^{3} u(x, t)\right|_{t=T, x \in \Omega}=0 .
$$

Boundary Value Problem III: Find a function $u(x, t)$ that is a solution to equation (1) in $Q$ that satisfies conditions (2) and also the condition

$$
\left.u(x, t)\right|_{t=0, x \in \Omega}=\left.D_{t}^{2} u(x, t)\right|_{t=0, x \in \Omega}=\left.D_{t} u(x, t)\right|_{t=0, x \in \Omega}=\left.D_{t}^{3} u(x, t)\right|_{t=0, x \in \Omega}=0 .
$$

Boundary Value Problem I is a usual initial boundary value problem for nonstationary differential equations of the fourth order (with respect to time). Boundary Value Problem II is a modified V. N. Vragov's problem (see [12-14]) for fourth-order quasihyperbolic equations. Finally, Boundary Value Problem III is in fact an elliptic boundary value problem.

In the present article, we propose sufficient conditions on the coefficients of (1) new compared to the previous works that guarantee the existence and uniqueness of regular solutions to boundary value problems I, II, or III.

\section{Solvability of boundary value Problems I-III}

Theorem 1. Suppose the fulfilment of the conditions

$$
\begin{gathered}
a^{i j, k}(x) \in C^{1}(\bar{\Omega}), \quad i, j=1, \ldots, n, \quad a_{0, k}(x) \in C(\bar{\Omega}), \quad k=0,1,2 ; \\
a^{i j, 3}(x) \in C^{2}(\bar{\Omega}), \quad a^{i j, 3}(x)=a^{j i, 3}(x), \quad i, j=1, \ldots, n, \quad a_{0,3}(x) \in C(\bar{\Omega}), \\
-a^{i j, 3}(x) \xi_{i} \xi_{j} \geqslant m_{0}|\xi|^{2}, \quad m_{0}>0, \quad x \in \bar{\Omega}, \quad \xi \in \mathbb{R}^{n} .
\end{gathered}
$$

Then, for every function $f(x, t)$ in $L_{2}(Q)$, Boundary Value Problem I has a solution $u(x, t)$ such that $D_{t}^{k} u(x, t) \in L_{2}\left(0, T ; W_{2}^{2}(\Omega) \cap \stackrel{\circ}{W} \underset{2}{1}(\Omega)\right), k=0,1,2,3, D_{t}^{4} u(x, t) \in L_{2}(Q)$. 
Proof. Make use of the method of continuation in a parameter. Let $\lambda \in[0,1]$. Consider the following problem: Find a function $u(x, t)$ that is a solution to the equation

$$
D_{t}^{4} u+A_{3} D_{t}^{3} u+\lambda \sum_{k=0}^{2} A_{k} D_{t}^{k} u=f(x, t)
$$

and $Q$ that satisfies conditions (2) and (3). Note that, for $\lambda=0$, this problem has a solution $u(x, t)$ belonging to the desired class; this follows from the fact that, for $\lambda=0$, equation (9) is a usual parabolic equation with respect to $u_{t t t}(x, t)$. Furthermore, by the theorem on the method of extension in a parameter (see [15, Chapter III, Sec. 14], the boundary value problem (9), (2), (3) has a regular solution $u(x, t)$ if $f(x, t) \in L_{2}(Q)$ and problem (9), (2), (3) is solvable in the class of regular solutions for $\lambda=0$ if all derivatives occurring in (9) are uniformly bounded in $L_{2}(Q)$.

For proving the desired boundedness, let us first consider the equality

$$
\int_{0}^{t} \int_{\Omega}\left[D_{\tau}^{4} u+A_{3} D_{\tau}^{3} u+\lambda \sum_{k=0}^{2} A_{k} D_{\tau}^{k} u\right] D_{\tau}^{3} u d x d \tau=\int_{0}^{t} \int_{\Omega} f D_{\tau}^{3} u d x d \tau
$$

Integrating by parts, applying Young's inequality and the inequality

$$
\int_{\Omega} w^{2}(x, t) d x \leqslant T \int_{0}^{t} \int_{\Omega} w_{\tau}^{2}(x, \tau) d x d \tau
$$

which is valid for functions $w(x, t)$ vanishing for $t=0$, and using conditions (6)-(8) and Gronwall's lemma, it is not hard to obtain from (10) the estimate

$$
\int_{\Omega}\left[D_{t}^{3} u(x, t)\right]^{2} d x+\sum_{i=1}^{n} \int_{0}^{t} \int_{\Omega}\left(D_{\tau}^{3} u_{x_{i}}\right)^{2} d x d \tau \leqslant C_{1} \int_{Q} f^{2} d x d t
$$

where the constant $C_{1}$ is defined only by the functions $a^{i j, k}(x), i, j=1, \ldots, n, a_{0, k}(x)$, $k=0,1,2,3$, and the number $T$.

Now, consider the equality

$$
-\int_{0}^{t} \int_{\Omega}\left(D_{\tau}^{4} u+A_{3} D_{\tau}^{3} u+\lambda \sum_{k=0}^{2} A_{k} D_{\tau}^{k} u\right) A_{3} D_{\tau}^{3} u d x d \tau=-\int_{0}^{t} \int_{\Omega} f A_{3} D_{\tau}^{3} u d x d \tau .
$$

Integrating by parts once again, applying Young's inequality, inequality (11), estimate (12), conditions (6)-(8), and also the second main inequality for elliptic operators (see [10, Chapter III, Stc. 8], and Gronwall's lemma, we conclude that solutions $u(x, t)$ to the boundary value problem (9), (2), (3) satisfy the second a priori estimate

$$
\sum_{i=1}^{n} \int_{\Omega}\left[D_{t}^{3} u_{x_{i}}(x, t)\right]^{2} d x+\sum_{i, j=1}^{n} \int_{0}^{t} \int_{\Omega}\left(D_{\tau}^{3} u_{x_{i} x_{j}}\right)^{2} d x d \tau \leqslant C_{2} \int_{Q} f^{2} d x d t
$$

where the constant $C_{2}$ is defined only by the functions $a^{i j, k}(x), a_{0, k}(x), i, j=1, \ldots, n$, $k=0,1,2,3$, the domain $\Omega$, and the number $T$.

Estimates (12) and (13) imply the obvious third estimate

$$
\int_{0}^{t} \int_{\Omega}\left(D_{\tau}^{4} u\right)^{2} d x d \tau \leqslant C_{3} \int_{Q} f^{2} d x d t
$$


of solutions $u(x, t)$ to the boundary value problem $(9),(2),(3)$; the constant $C_{3}$ in this estimate is again defined only by the functions $a^{i j, k}(x), a_{0, k}(x), i, j=1, \ldots, n, k=0,1,2,3$, the domain $\Omega$, and the number $T$.

Estimates (12)-(14) give the desired uniform boundedness over $\lambda$ in $L_{2}(Q)$ of all derivatives occurring in (9). As we already said above, this boundedness and the solvability of the boundary value problem $(9),(2),(3)$ for $\lambda=0$ give the solvability of this problem in the desired class also for $\lambda=1$. This exactly means the validity of the theorem.

The theorem is proved.

Before proving the following theorem on the solvability of Problem I in the class of regular solutions, we formulate an auxiliary assertion on the nonnegativity of the scalar product of a pair of second-order differential operators.

Let $A$ and $B$ be differential operators whose action is defined by the equality

$$
\begin{aligned}
& A v=\frac{\partial}{\partial x_{i}}\left(a^{i j}(x) v_{x_{j}}\right)+a_{0}(x) v, \\
& B v=\frac{\partial}{\partial x_{i}}\left(b^{i j}(x) v_{x_{j}}\right)+b_{0}(x) v .
\end{aligned}
$$

Proposition 1. Suppose the fulfillment of the conditions

$$
\begin{gathered}
a^{i j}(x) \in C^{2}(\bar{\Omega}), \quad b^{i j}(x) \in C^{2}(\bar{\Omega}), \quad a^{i j}(x)=a^{j i}(x), \quad b^{i j}(x)=b^{j i}(x), \quad x \in \bar{\Omega}, \quad i, j=1, \ldots, n ; \\
a_{0}(x) \in C^{1}(\bar{\Omega}), \quad b_{0}(x) \in C^{1}(\bar{\Omega}), \quad a_{0}(x) \leqslant-a_{0}<0, \quad b_{0}(x) \leqslant-b_{0}<0, \quad x \in \bar{\Omega} ; \\
\exists \alpha^{i}(x): \alpha^{i}(x) \in C(\bar{\Omega}), \quad \alpha^{i}(x) \geqslant 0, \quad x \in \bar{\Omega}, \quad i=1, \ldots, n, \\
\alpha^{i}(x) \xi_{i}^{2} \leqslant a^{i j}(x) \xi_{i} \xi_{j} \leqslant M_{0} \alpha^{i}(x) \xi_{i}^{2}, \quad x \in \bar{\Omega}, \quad \xi \in \mathbb{R}^{n} ; \\
\left|a_{x_{k}}^{i j}(x)\right| \leqslant M_{1} \sqrt{\alpha^{i}(x)}, \quad x \in \bar{\Omega}, \quad i, j, k=1, \ldots, n ; \\
a^{i j}(x) \nu_{i} \nu_{j}=0 \quad \text { for } \quad x \in \Gamma ; \\
b^{i j}(x) \xi_{i} \xi_{j} \geqslant m_{0}|\xi|^{2}, \quad m_{0}>0, \quad x \in \bar{\Omega}, \quad \xi \in \mathbb{R}^{n} ; \\
{\left[a_{0}(x) b^{i j}(x)+b_{0}(x) a^{i j}(x)+\frac{1}{2}\left(a_{x_{k}}^{i j}(x) b^{k l}(x)\right)_{x_{l}}+\frac{1}{2}\left(b_{x_{k}}^{i j}(x) a^{k l}(x)\right)_{x_{l}}-\right.} \\
\left.-\left(a_{x_{k}}^{i l}(x) b_{x_{l}}^{j k}(x)\right)\right] \xi_{i} \xi_{j} \leqslant 0, \quad x \in \bar{\Omega}, \quad \xi \in \mathbb{R}^{n} ; \\
a_{0}(x) b_{0}(x)+\frac{1}{2}\left(a_{0 x_{i}}(x) b^{i j}(x)\right)_{x_{j}}+\frac{1}{2}\left(b_{0 x_{i}}(x) a^{i j}(x)\right)_{x_{j}} \geqslant 0, \quad x \in \bar{\Omega} .
\end{gathered}
$$

Then every function $v(x) \in W_{2}^{2}(\Omega) \cap \stackrel{\circ}{W}_{2}^{1}(\Omega)$ satisfies the inequality

$$
\int_{\Omega} A v B v d x \geqslant 0
$$

This assertion is proved in [16].

We say that operators $A$ and $B$ of the above form satisfy the $(A, B)$-condition if the coefficients of these operators satisfy all conditions of Proposition 1.

Theorem 2. Suppose the fulfillment of the $\left(-A_{3},-A_{2}\right)$-condition and also of the condition

$$
a^{i j, k}(x) \in C^{1}(\bar{\Omega}), \quad i, j=1, \ldots, n, \quad a_{0, k}(x) \in C(\bar{\Omega}), \quad k=0,1 .
$$

Then, for every function $f(x, t)$ such that $f(x, t) \in L_{2}(Q), f_{t}(x, t) \in L_{2}(Q), f(x, 0)=0$ for $x \in \bar{\Omega}$, Boundary Value Problem I has a solution $u(x, t)$ such that $D_{t}^{k} u(x, t) \in L_{\infty}\left(0, T ; W_{2}^{2}(\Omega) \cap\right.$ $\left.\stackrel{\circ}{W}_{2}^{1}(\Omega)\right), k=0,1,2,3, D_{t}^{4} u(x, t) \in L_{\infty}\left(0, T ; L_{2}(\Omega)\right)$. 
Proof. Observe first of all that the $\left(-A_{3},-A_{2}\right)$-condition in particular means that $-A_{3}$ is an elliptic-parabolic operator in $\bar{\Omega}$ and $-A_{2}$ is an elliptic operator.

Let $\varepsilon$ be a positive number. Define operators $A_{3, \varepsilon}$ and $L_{\varepsilon}$ :

$$
A_{3, \varepsilon}=A_{3}+\varepsilon A_{2}, \quad L_{\varepsilon}=L+\varepsilon A_{2} D_{t}^{3} .
$$

Consider the following boundary value problem: Find a function $u(x, t)$ that is a solution to the equation $L_{\varepsilon} u=f$ in $Q$ that satisfies conditions (2) and (3). Obviously, this boundary value problem is Boundary Value Problem I and that it satisfies all conditions of Theorem 1. Moreover, due to the condition $f(x, t) \in L_{2}(Q), f_{t}(x, t) \in L_{2}(Q)$, a solution $u(x, t)$ to this problem satisfies the memberships

$$
D_{t}^{k} u(x, t) \in L_{\infty}\left(0, T ; W_{2}^{2}(\Omega) \cap \stackrel{\circ}{W} \underset{2}{1}(\Omega)\right), \quad k=0,1,2,3,4, \quad D_{t}^{5} u(x, t) \in L_{2}(Q)
$$

(this fact stems from its validity for the "shortened" equation $D_{t}^{4} u+A_{3, \varepsilon} D_{t}^{3} u=f(x, t)$ and the corresponding a priori estimates).

Differentiate the equation $L_{\varepsilon} u=f(x, t)$ with respect to $t$ (this is possible due to memberships (16)), multiply it by $D_{t}^{4} u(x, t)$, and integrate it over the cylinder $\{x \in \Omega, \quad 0<\tau<t\}$. Involving the ellipticity of the operators $-A_{3, \varepsilon}$ and $-A_{2}$, applying Young's inequality, inequality (11), and Gronwall's lemma, we obtain the estimate

$$
\varepsilon \int_{0}^{t} \int_{\Omega}\left(A_{2} D_{\tau}^{4} u\right)^{2} d x d \tau+\sum_{i=1}^{n} \int_{\Omega}\left[D_{t}^{4} u_{x_{i}}(x, t)\right]^{2} d x+\int_{\Omega}\left[A_{2} D_{t}^{3} u(x, t)\right]^{2} d x \leqslant C_{4} \int_{Q} f_{t}^{2} d x d t
$$

where the constant $C_{4}$ is defined only by the functions $a^{i j, k}(x), a_{0, k}(x), i, j=1, \ldots, n, k=0,1$, and also the number $T$.

Let $\left\{\varepsilon_{m}\right\}_{m=1}^{\infty}$ be a sequence of positive numbers converging to zero and let $\left\{u_{m}(x, t)\right\}_{m=1}^{\infty}$ be a sequence of solutions to the equation $L_{\varepsilon_{m}} u=f$ satisfying (2) and (3). Estimate (17), the second main inequality for elliptic operators, and the reflexivity of a Hilbert space mean that there exists a sequence $\left\{u_{m_{l}}(x, t)\right\}_{l=1}^{\infty}$ and a function $u(x, t)$ that satisfy the following weak convergences as $l \rightarrow \infty$ in $L_{2}(Q)$ :

$$
\begin{gathered}
\varepsilon_{m_{l}} A_{2} D_{t}^{3} u(x, t) \rightarrow 0, \\
D_{t}^{4} u_{m_{l}}(x, t) \rightarrow D_{t}^{4} u(x, t), \\
A_{k} D_{t}^{k} u_{m_{l}}(x, t) \rightarrow A_{k} D_{t}^{k} u(x, t), \quad k=0,1,2,3 .
\end{gathered}
$$

Obviously, the limit function $u(x, t)$ is a solution to Boundary Value Problem I and this solution still satisfies (17). Therefore, the function $u(x, t)$ is the desired solution to the problem under study.

The theorem is proved.

Turn to investigating the solvability of Boundary Value Problem II.

The main difference of Boundary Value Problem II from Boundary Value Problem I is that, in its study, it is impossible to use Gronwall's lemma. Gronwall's lemma can be replaced by smallness conditions.

We will give the simplest version of the theorem in the solvability of a Boundary Value Problem II, whose prove involves smallness conditions.

Let operators $A_{0}$ and $A_{1}$ be defined with the use of the parameter $\beta$ and the operators $\widetilde{A}_{0}$ and $\widetilde{A}_{1}$ :

$$
A_{0}=\beta \widetilde{A}_{0}, \quad A_{1}=\beta \widetilde{A}_{1}, \quad \widetilde{A}_{k}=\frac{\partial}{\partial x_{i}}\left(\widetilde{a}^{i j, k}(x) \frac{\partial}{\partial x_{j}}\right)+\widetilde{a}_{0 i}(x), \quad k=0,1 .
$$


Theorem 3. Suppose the fulfillment of the conditions

$$
\begin{gathered}
a^{i j, k}(x) \in C^{2}(\bar{\Omega}), \quad a^{i j, k}(x)=a^{j i, k}(x), \quad i, j=1, \ldots, n, \quad k=2,3 ; \\
\widetilde{a}^{i j, k}(x) \in C^{1}(\bar{\Omega}), \quad i, j=1, \ldots, n, \quad \widetilde{a}_{0, k}(x) \in C(\bar{\Omega}), \quad k=0,1 ; \\
a^{i j, k}(x) \xi_{i} \xi_{j} \geqslant m_{0}|\xi|^{2}, \quad m_{0}>0, \quad x \in \bar{\Omega}, \quad \xi \in \mathbb{R}^{n}, \quad k=2,3 ; \\
a_{0, k}(x) \in C(\bar{\Omega}), \quad k=0,1,2,3, \quad a_{0, k}(x) \leqslant 0, \quad k=2,3 .
\end{gathered}
$$

Then there exists a positive number $\beta_{0}$ such that for $|\beta|<\beta_{0}$ and $f(x, t) \in L_{2}(Q)$, Boundary Value Problem II has a solution $u(x, t)$ such that $D_{t}^{k} u(x, t) \in L_{2}\left(0, T ; W_{2}^{2}(\Omega) \cap \stackrel{\circ}{W} \underset{2}{1}(\Omega)\right)$, $k=0,1,2,3, D_{t}^{4} u(x, t) \in L_{2}(Q)$.

Proof. For $\lambda=0$, Boundary Value Problem II for equation (9) has a solution $u(x, t)$ in the desired class; this follows from the fact that for $\lambda=0$ equation (9) is an inverse parabolic equation with respect to $D_{t}^{3} u(x, t)$. Further, consider (10). Integrating by parts and estimating the last two summands on the left-hand side (10) from above with the use of (11), we infer that there exists a positive number $\beta_{1}$ such that for $|\beta|<\beta_{0}$ we have the a priori estimate

$$
\sum_{i=1}^{n} \int_{Q}\left(D_{t}^{3} u_{x_{i}}\right)^{2} d x d t \leqslant C_{5} \int_{Q} f^{2} d x d t
$$

with the constant $C_{5}$ defined only by the coefficients of the operators $A_{k}, k=0,1,2,3$.

At the next step, consider the equality

$$
\int_{Q}\left[D_{t}^{4} u+A_{3} D_{t}^{3} u+\lambda \sum_{k=0}^{2} A_{k} D_{t}^{k} u\right] A_{2} D_{t}^{3} u d x d t=\int_{Q} f A_{2} D_{t}^{3} u d x d t .
$$

Reckoning with the ellipticity of $A_{2}$ and $A_{3}$ and using the second main inequality for a pair of elliptic operators [10, Chapter III, Sec. 8], it is not hard to show that there exists a number $\beta_{0}$ such that $0<\beta_{0} \leqslant \beta_{1}$, and for $|\beta|<\beta_{0}$, for solutions $u(x, t)$ to Boundary Value Problem II for equation (9), estimate (13) holds with some constant $C_{6}$ on the right-hand side that is defined only by the coefficients of the operators $A_{k}, k=0,1,2,3$, and the domain $\Omega$.

Estimate (14) with the corresponding constant $C_{7}$ on the right-hand side obviously follows from the previous estimates.

The obtained estimates of solutions to Boundary Value Problem II for equation (9) and the theorem on the method of continuation in a parameter and give the solvability of Boundary Value Problem II for equation (1) in the desired class.

The theorem is proved.

Theorem 4. Suppose the fulfilment of the conditions

$$
\begin{gathered}
a^{i j, k}(x) \in C^{2}(\bar{\Omega}), \quad a^{i j, k}(x)=a^{j i, k}(x), \quad a_{0, k}(x) \in C(\bar{\Omega}), \quad i, j=1, \ldots, n, \quad k=0,1,2,3 ; \\
a^{i j, k}(x) \xi_{i} \xi_{j} \geqslant m_{0}|\xi|^{2}, \quad m_{0}>0, \quad x \in \bar{\Omega}, \quad \xi \in \mathbb{R}^{n}, \quad a_{0, k}(x) \leqslant 0, \quad k=2,3 ; \\
-a^{i j, k}(x) \xi_{i} \xi_{j} \geqslant m_{1}|\xi|^{2}, \quad m_{1}>0, \quad x \in \bar{\Omega}, \quad \xi \in \mathbb{R}^{n}, \quad a_{0, k}(x) \geqslant 0, \quad k=0,1 ; \\
A_{0}=\beta \widetilde{A}_{0} .
\end{gathered}
$$

Then there is a positive number $\beta_{0}$ such that, for $|\beta|<\beta_{0}$ and $f(x, t) \in L_{2}(Q)$, Boundary Value Problem III has a solution $u(x, t)$ such that $D_{t}^{k} u(x, t) \in L_{2}\left(0, T ; W_{2}^{2}(\Omega) \cap \stackrel{\circ}{W} \frac{1}{2}(\Omega)\right), k=0,1,2,3$, $D_{t}^{4} u(x, t) \in L_{2}(Q)$. 
Proof. Show that solutions $u(x, t)$ to Boundary Value Problem III of the class mentioned in the statement of the theorem satisfy the desired a priori estimates.

Multiply equation (1) by $D_{t}^{2} u(x, t)$. Integrating over $Q$, applying integration by parts, and using (20)-(22), it is not hard to obtain the first a priori estimate for solutions $u(x, t)$ to Boundary Value Problem III:

$$
\int_{Q}\left[\left(D_{t}^{3} u\right)^{2}+\sum_{i=1}^{n}\left(D_{t}^{2} u_{x_{i}}\right)^{2}\right] d x d t \leqslant C_{8} \int_{Q} f^{2} d x d t
$$

here the constant $C_{8}$ is defined only by the coefficients of the operators $A_{k}, k=0,1,2,3$.

At the next step, multiply equation (1) by $A_{2} D_{t}^{3} u(x, t)$ and integrate it over $Q$. Using conditions (20)-(23), inequality (11), and also the second main inequality for a pair of elliptic operators, we conclude that there exists a number $\beta_{0}$ such that for $|\beta|<\beta_{0}$ we have a second estimate

$$
\sum_{i, j=1}^{n} \int_{Q}\left(D_{t}^{3} u_{x_{i} x_{j}}\right)^{2} d x d t \leqslant C_{9} \int_{Q} f^{2} d x d t
$$

with the constant $C_{9}$ defined only by the coefficients of the operators $A_{k}, k=0,1,2,3$, and the domain $\Omega$.

The last a priori estimate

$$
\int_{Q}\left(D_{t}^{4} u\right)^{2} d x d t \leqslant C_{10} \int_{Q} f^{2} d x d t
$$

obviously stems of the previous two estimates.

Using estimates (24)-(26) and the method of continuation in a parameter (for example, with the use of the equation

$$
\left.D_{t}^{4} u+A_{2} D_{t}^{2} u+\lambda\left(A_{3} D_{t}^{3} u+A_{1} D_{t} u+A_{0} u\right)=f(x, t)\right),
$$

it is not hard to obtain the desired solvability of Boundary Value Problem III.

The theorem is proved.

\section{Conclusion.}

Observe first of all that the conditions of Proposition 1 are fulfilled, for instance, if the numbers $a_{0}$ and $b_{0}$ are large.

Furthermore, it is not hard to generalize the obtained results to equations more general than (1); for example, to equations with general second-order elliptic operators $A_{k}$.

Some of the conditions of the proven theorems can be changed: for example, we can discard the sign-definiteness of the operator $A_{0}$ from Theorem 4.

Observe finally that conditions (18) and (23) mean that $\widetilde{A}_{1}$ and $\widetilde{A}_{0}$ are fixed operators, whereas the number $\beta$ is a parameter (namely, a smallness parameter).

The work of the author was carried out in the framework of the State Contract of the Sobolev Institute of Mathematics (Project 0314-2019-0010).

\section{References}

[1] S.L.Sobolev, On a boundary value problem of mathematical physics, Izv. Academy of Sciences of the USSR. Ser. Mat., 18(1954), no. 2, 3-50 (in Russian). 
[2] G.V.Demidenko, S.V.Uspensky, Equations and systems not resolved with respect to the highest derivative, Novosibirsk, Scientific, 1998 (in Russian).

[3] A.I.Kozhanov, Composite Type Equations and Inverse Problems, Utrecht, VSP, 1999.

[4] I.E.Egorov, S.G.Pyatkov, S.V.Popov, Non-classical differential-operator equations, Novosibirsk, Science, 2000 (in Russian).

[5] S.G.Pyatkov, Operator Theory. Nonclassical Problems, Utrecht, Boston, Koln, Tokyo: VSP, 2002.

[6] G.A.Sviridyuk, V.E.Fedorov, Linear Sobolev Type Equations and Degenerate Semigroup of Operators, Utrecht: VSP, 2003.

[7] A.G.Sveshnikov, A.B.Alshin, M.O.Korpusov, Yu.D.Pletner, Linear and nonlinear equations of the Sobolev type, Moscow, Fizmatlit, 2007 (in Russian).

[8] V.I.Zhegalov, A.N.Mironov, E.A.Utkina, Equations with a dominant partial derivative, Kazan, Kazan Federal University, 2014 (in Russian).

[9] S.L.Sobolev, Some applications of functional analysis in mathematical physics, Science, Moscow, 1988 (in Russian).

[10] O.A.Ladyzhenskaya, N.N.Uraltseva, Linear and quasilinear equations of elliptic type, Moscow, Nauka, 1973 (in Russian).

[11] H.Triebel, Interpolation Theory. Function Spaces. Differential Operators, VEB Deutcher Verlag der Wissenschaften, Berlin, 1978.

[12] V.N.Vragov, On the theory of boundary value problems for mixed-type equations, Differential Equations, 13(1977), no. 6, 1098-1105.

[13] I.E.Egorov, V.E.Fedorov, Non-classical equations of high-order mathematical physics, Publishing house of the Computing Center SB RAS, Novosibirsk, 1995 (in Russian).

[14] A.I.Kozhanov, N.R.Pinigina, Boundary problems for some non-classical differential equations, Math. Notes, 101(2017), no. 3, 467-474. DOI: 10.1134/S0001434617030087

[15] V.A.Trenogin, Functional analysis, Moscow, Nauka, 1980.

[16] A.I.Kozhanov, Linear inverse problems for a class of degenerate equations of Sobolev type, Vestn. SUSU. Ser. Mat. modeling and programming, 264(2012), no. 5, issue 11, 33-42.

\title{
Краевые задачи для уравнений соболевского типа четвертого порядка
}

\author{
Александр И. Кожанов \\ Институт математики им. С. Л. Соболева СО РАН \\ Novosibirsk, Российская Федерация
}

\begin{abstract}
Аннотация. Целью статьи является исследование разрешимости в пространствах Соболева краевых задач для некоторых классов линейных уравнений четвертого порядка соболевского типа. Докажем, что начально-краевые задачи с данными как в начальный момент времени, так и в конечные моменты времени могут быть корректными для исследуемых уравнений.
\end{abstract}

Ключевые слова: дифференциальное уравнение четвертого порядка соболевского типа, краевая задача, существование, единственность. 